\title{
La investigación como camino para la actualización permanente del conocimiento
}

\author{
Gerardo Cardozo-Rincón*
}

* Doctor en Filosofía y Lingüística, Universidad Autónoma de Madrid, España. Docente investigador, Universidad Pedagógica y Tecnológica de Colombia, Tunja, Colombia.

Correo electrónico:

gerardo8202@hotmail.com

Recibido: 10 de marzo del 2014 Aprobado: 06 de noviembre del 2014

Cómo citar este artículo: CardozoRincón, Gerardo. "La investigación como camino para la actualización permanente del conocimiento". Rastros Rostros 16.30 (2014): 89-94. Impreso. doi: http://dx.doi. org/10.16925/ra.v16i30.823

\section{Resumen}

En este artículo se presentan algunos aspectos que tienen que ver con la investigación en el ámbito académico. En primer lugar, se plantea la imposibilidad de la objetividad como un reto que deban asumir los investigadores; en este caso, se muestra como un aspecto que aumenta el compromiso de los investigadores. Posteriormente, se destacan los roles de quienes intervienen en una investigación académica. Se observa el papel del orientador y se mencionan algunas de las posibilidades que tienen los jurados frente a los trabajos investigativos de los tesistas. Esto lleva a que el final sea ejemplificado con lo que podría suceder con la evaluación de los informes finales de investigación.

Palabras clave: actualización del conocimiento, investigación, objetividad, paradigmas.

\section{Research as a Path for the Constant Updating of Knowledge}

\section{Abstract}

This article presents certain aspects of research in the academic setting. First, it puts forward the impossibility of objectivity being a challenge that researchers must meet; here it is shown as a factor that increases researchers' commitment. Following this, the roles of those who are involved in academic research are highlighted. The part played by the tutor is examined, and mention is made of some options available to judges when looking at the research work of students presenting their theses. The final part of this article offers some examples of what can occur in the evaluation of final research reports.

Keywords: updating of knowledge, research, objectivity, paradigms.

\section{A pesquisa como caminho para a atualização permanente do conhecimento}

\section{Resumo}

Este artigo apresenta alguns aspectos relacionados com a pesquisa no âmbito acadêmico. Em primeiro lugar, expõe-se a impossibilidade da objetividade ser um desafio que os pesquisadores devam assumir; neste caso, mostra-se como um aspecto que aumenta o compromisso dos pesquisadores. Posteriormente, destacam-se os papéis de quem intervém em uma pesquisa acadêmica. Observa-se o papel do orientador e mencionam-se algumas das possibilidades que os jurados têm ao abordarem os trabalhos de pesquisa dos estudantes. Isso leva a que o final seja exemplificado com o que poderia acontecer com a avaliação dos relatórios finais de pesquisa.

Palavras-chave: atualização do conhecimento, pesquisa, objetividade, paradigmas. 


\section{Introducción}

Es innegable que una de las características del conocimiento científico debe ser la objetividad. Siempre se espera que cualquier investigación ofrezca algún aporte al ámbito en el que esté inscrita; esto implica que el investigador debe ser riguroso tanto en los métodos como en la presentación de resultados. Sin embargo, el miedo a salirse de los parámetros objetivos muchas veces opaca la experiencia, las perspectivas, la capacidad de análisis $y$, ante todo, la verdadera propuesta de un investigador.

Es común encontrar que cuando un investigador expone alguna idea o refuta otras conocidas, un lector espera las fuentes de esa propuesta; esto equivale a decir que solamente es válido el conocimiento presentado por autores - muchas veces, clásicos- y se tomará como una injuria o un irrespeto académico el contradecir a tales autores. En fin, esto limita la toma de posición del investigador a la hora de analizar información y presentar resultados.

Una preocupación surge cuando un trabajo investigativo es rechazado porque los datos presentados o las conclusiones no concuerdan con lo dicho por los autores de décadas pasadas. Aunque sea algo paradójico, en este caso sería ideal recordar una fuente clásica que, de alguna forma, nos exhorta y abre las puertas para que discutamos y replanteemos las posturas de los autores que para muchos son irrefutables (por no repetir "clásicos"): "La ciencia carece de axiomas evidentes; incluso los principios más generales y seguros son postulados que pueden ser corregidos o reemplazados. ... Tan pronto como ha sido establecida una teoría científica, corre el riesgo de ser refutada o, al menos, de que se circunscriba su dominio" (Bunge 33).

\section{El investigador}

Es pertinente la metáfora ya bien conocida de que si se afirma que la tierra es redonda, nos quemarán. Por eso, el papel de todos los implicados en la investigación científica va de la mano con el compromiso de reconocer que la teoría puede replantearse e ir cambiando. La experiencia de un investigador, en este sentido, no se mediría por la cantidad de información que conozca y pueda repetir, sino por su capacidad para enfrentarse al conocimiento ya existente, por medio de procesos investigativos que le permitan reafirmar o alimentar un área del saber. Es importante anotar que aquello que aparece en los libros de autores clásicos, más allá de constituirse como fuentes de conocimiento obligatorio, suele ser un limitante cuando estos se toman como indiscutibles. Por eso, la visión de los investigadores debe llevar a reconocer que los autores tradicionales también creen, opinan, observan y experimentan realidades, y eso es lo que comparten en sus textos.

No se debe concebir al investigador como un ser que solamente recopila, toma teorías, confronta lo recopilado con las teorías y presenta conclusiones. Esto hace que se desconozca el papel del investigador como una persona con ideas, que piensa y analiza más allá de la fundamentación teórica que haya necesitado durante el proceso. El investigador debe llegar a un resultado que quizá no coincida con el que se esperaba al inicio del proceso, pero que sí dé cuenta de una interacción con la realidad a la que le puede aportar algo nuevo. También es importante que los estudiantes se enfrenten a situaciones no planificadas en sus investigaciones, pues esto los ejercitará en lo que respecta a la toma de decisiones. Ante esta realidad, Mora (359), al hablar sobre la importancia de que los niños y jóvenes aprendan a investigar, asegura que

lo que es innegable, es que en la creatividad del ser humano ha habido y seguirán habiendo respuestas para muchos interrogantes y problemas. Los grandes problemas del mundo no se van a resolver solos; los tendrá que resolver el hombre, y podrá hacerlo en la medida en que sepa observar, analizar e interpretar las variables de su entorno. Y no solo eso, una vez logrado lo anterior, asimismo tendrá que saber tomar las decisiones. Lo cual también es una habilidad fundamental que tiene que adquirir.

La responsabilidad que se le asigna al hombre para solucionar los problemas del mundo implica que se arriesgue a tomar decisiones que nazcan de su creatividad y del ingenio para hallar respuestas. No se trata de gritar "eureka" en un segundo, sino de arriesgarse por ideas que, aunque no sean las compartidas por una mayoría, puedan arrojar datos nuevos acerca de los problemas que se quieran solucionar. Cuando hablamos de datos nuevos, no nos referimos a unas conclusiones que se presentarían en un informe final, sino a toda la realidad susceptible de descripción que se encuentre en el recorrido investigativo.

\section{El proceso de investigación}

Se requiere que el proceso investigativo no se asuma como una línea con un punto inicial, un punto medio 
protagonizado por una metodología y unas conclusiones que se espera que se adecúen al conocimiento compartido por muchos o, por lo menos, los expertos en el tema. Ese recorrido exige el toque de sensibilidad propia de la humanidad ante cualquier situación mundana. Tena (488) comenta que

el investigador cualitativo requiere contar con una gran capacidad para interpretar toda la información recopilada en el campo de investigación, esto más que una técnica es un arte, que no consiste solo en el análisis frío de los datos obtenidos, sino en una descripción sensible y detallada de estos.

Es claro que al hablar de interpretación, idealizamos a un hermeneuta que quiere encontrar algo en su enfrentamiento con los datos; es alguien que no quiere limitarse a describir o analizar los datos sin que su papel como investigador se justifique. De ser así, ese proceso lo podría desarrollar cualquiera - con o sin formación investigativa - y los resultados serían los mismos.

En relación con la interpretación de los análisis, Camacho (171) dice que "su importancia está en que permite al investigador hacer sus aportes personales y dar sentido a lo analizado". Sobra aclarar que esos aportes personales no se refieren a opiniones o a la manifestación de gustos o caprichos. Estos posibilitan el autoreconocimiento del investigador como el responsable de una interpretación que exige que esté preparado para enfrentarse a la realidad y al problema identificado. Son aportes personales en la medida en que se espera que el investigador tenga ese toque especial, de tal forma que su proceso investigativo lo pueda fortalecer para uno siguiente. En el mismo sentido, los aportes del investigador son necesarios, pues de no ser así, por miedo a desatarse de la objetividad, el papel del investigador sería nulo o se extendería la idea de que la investigación no requiere ideas, pensamiento, propuestas, sino solamente la traducción de hechos a palabras.

En un plano más específico, como el de la investigación lingüística, es común encontrar dificultades a la hora de analizar la información. Es lo que sucede, por ejemplo, en estudios pragmáticos enfocados a la identificación del significado de los enunciados realizados por el hablante. En estos casos, se concibe la idea de una interpretación estrictamente objetiva que dé cuenta de las intenciones comunicativas de los hablantes. Sin embargo, de manera cruda podemos afirmar que esa objetividad es una ensoñación, pero no un obstáculo. Por el contrario, si se acepta que es imposible un nivel perfecto de objetividad, el investigador verá la necesidad de profundizar en estudios pragmáticos que lo capaciten para que el análisis y la interpretación sean satisfactorios. Así, el investigador estará más comprometido con el trabajo que realice y se sentirá en la obligación de corroborar su papel en cada una de las situaciones que encuentre en su recorrido investigativo. En palabras de Beuchot:

La hermenéutica, surgida de la filosofía, trata de interpretar el significado de un texto, pero sabe que no logrará apresar de manera completamente objetiva el mensaje del autor, porque en la transacción interpretativa se introducirá la subjetividad del lector o intérprete, del hermeneuta. En cambio, la pragmática, disciplina que configura una rama de la semiótica, pretende confiar en que se puede lograr la objetividad y rescatar el significado del autor (el speaker's meaning), esto es, la intencionalidad del hablante o autor de un texto, y no solo la intencionalidad subjetiva del hermeneuta. (8)

Cabría resaltar que la pragmática pretende confiar en el alcance de la objetividad; en efecto, existe la posibilidad de que un estudio pragmático le atine a la identificación de la intencionalidad de un emisor, lo que seguramente llevaría al reconocimiento del significado del hablante del enunciado. No obstante, también existe la posibilidad de que se asigne arbitrariamente una posible intención comunicativa al hablante y se consideraría como un significado del hablante algo que no corresponde a lo que realmente el emisor quiso decir. Lo curioso es que en ambos casos, tanto en la identificación adecuada como en la falla al identificar el significado pragmático, podría hablarse de objetividad por parte del investigador, si se considera que no quiere involucrarse ni aportar nada distinto de lo que se ha propuesto dar a conocer por medio de un análisis. La diferencia es que, en el primer caso, acertaría y, en el segundo, fallaría. ¿De qué depende? La respuesta a esta pregunta conlleva que se demerite el rol apoteósico de la objetividad en los análisis y en la interpretación, pues estos dos pasos dependen del investigador como tal. Aunque un investigador quiera ser inigualablemente objetivo en sus trabajos, si no se ha preparado lo suficiente en lo que respecta al tema, sino que pretende conseguir buenos resultados a punta de "buenas" intenciones, lo más seguro es que fracase. Por otra parte, un investigador que conozca bien el tema sabrá, verbigracia, que el estudio del significado del hablante depende de un contexto, de las características del 
emisor, del conocimiento compartido, de la intención comunicativa, de la distancia proxémica entre los interlocutores y, en general, de todos los elementos situacionales del discurso (unidireccional o interaccional); de ser así, el investigador estará en capacidad de incluir sus aportes personales con respecto al análisis, al proceso investigativo, al tema como tal y, especialmente, al enriquecimiento de las teorías existentes que, sin lugar a dudas, será algo que lo llene de placer.

Hasta este punto, se han planteado las bases para sostener que una investigación permite la actualización del conocimiento y un enfrentamiento científico con los clásicos. Es común escuchar que se busca fomentar la actitud crítica de los estudiantes en todos los niveles de formación; sin embargo, existe la posibilidad de que si un discente presenta una idea que refuta a los clásicos, eso pueda tomarse como un acto caprichoso. Por tal motivo, es necesario que se generalice una postura sobre la investigación que excluya definitivamente al tradicionalista acérrimo, para quien lo más odioso y tedioso es la actualización y el reconocimiento de que podrá existir algo distinto de lo que leyó o le enseñaron hace unas cuantas décadas. No en vano, Bunge (41) comenta que "el conocimiento científico es a veces desagradable, a menudo contradice a los clásicos (sobre todo si es nuevo)".

\section{La investigación en la academia}

En el ámbito educativo, especialmente en educación superior, los estudiantes deben enfrentarse a un reto académico protagonizado por la investigación. En este punto, es ineludible mencionar el papel de los directores y los jurados de los trabajos de grado. El director es quien guía al estudiante investigador en la búsqueda de un conocimiento nuevo o en la reafirmación de lo ya conocido. Los jurados son los llamados a valorar esos productos investigativos.

De manera hipotética, puede pensarse en un estudiante investigador que establece como objetivo conocer los resultados de la aplicación de unas técnicas especiales para mejorar ortografía en un grupo de estudiantes; sin duda, considerará la posibilidad de que al final muestre los textos elaborados por los mejores discentes. Ante situaciones como estas, los implicados en la orientación y en la evaluación de las investigaciones deben evitar que se fortalezca la falacia de que una buena investigación es la que siempre muestra resultados favorables. Si en la realidad esas técnicas no mejoraran en nada la ortografía, el estudiante investigador no debe tener miedo de una baja calificación por parte de los jurados, pues ellos valoran el proceso de investigación sin tener la limitación de que se obtenga un resultado particular, sino con la mirada puesta en los datos que provengan únicamente del ejercicio investigativo. De esta manera, se reconocería un aporte, puesto que una investigación de ese talante ya enseñaría que por ese lado no es, que esas técnicas no sirven para mejorar ortografía, y esto representa un avance significativo para pensar en otras técnicas.

Benites (169) afirma que: "se requiere que los estudiantes que inician un proyecto de investigación aborden problemas de sus propios países, regiones o ciudades, y que lo hagan de manera creativa y sin ninguna restricción". Alguien inocentemente podría asegurar que la propuesta de Benites no es apropiada en la medida en que se requieren restricciones de tipo metodológico, en general, que no se puede dejar que el estudiante haga lo que caprichosamente desee y sin fundamentos. Es innegable que hay unos requerimientos metodológicos para toda investigación; sin embargo, Benites se refiere más que todo a las restricciones que podrían sobreponerse a la creatividad de los estudiantes. Se busca que no haya limitaciones en sus ideas, sino más bien que se les permita jugar con la curiosidad. En estos casos, el papel del docente no consiste en decirles a los estudiantes algo como "van a desarrollar un proyecto de investigación sobre ... y con esta metodología", sino que propenda hacia el desarrollo del alumno como un ser consciente de que si algo le genera curiosidad, es digno de estudiarse, especialmente si ese algo tiene relación con un problema identificable en la realidad que lo circunda.

Con base en lo anterior, el papel del profesor implica que se muestre el conocimiento como un soporte teórico relevante en la formación académica y, a la vez, como susceptible de discusión y de renovarse constantemente. Por eso, es importante tener en cuenta la posición de Cruz (344): "Los profesores deben infundir en los alumnos la importancia de la investigación en el terreno académico y en el profesional, destacando su relevancia tanto en la generación de conocimiento como en la búsqueda de soluciones a problemas". Por lo tanto, las investigaciones no pueden verse obstaculizadas por las teorías de los autores clásicos que no abordaron los mismos problemas. En una investigación se pueden seguir caminos parecidos a los que siguieron los clásicos, así como distintos, pues la idea no es repetir los procedimientos tradicionales sólo porque así debe ser, sino según si estos constituyen el mejor camino que 
contribuya al logro particular de cada investigación. En este sentido, la metodología también es susceptible de adecuación, pues no es lógico que se reconozca cada investigación como única, pero que todas se ciñan a unos pasos o a modelos impuestos más que todo por la tradición y la costumbre. De hecho, se puede afirmar que la elaboración de los marcos metodológicos suele verse muy limitada por algunos paradigmas propuestos hace varias décadas.

La fundamentación teórica es imprescindible en una investigación; no obstante, esto no se contrapone a la idea de que la realidad también es un soporte que puede mencionarse. Por lo tanto, el investigador, estudiante o no, puede valerse del conocimiento que tiene de la realidad compartida sin que tenga que recurrir a un autor que diría lo mismo. Lo que se busca con esta idea es que el investigador sea reconocido no solo como protagonista del proceso, sino también como un autor de los planteamientos que aparecen en el informe y que no son tomados de otros autores. Esto lleva a que se piense que preguntas como "¿y esto quién lo dijo?, ¿de dónde lo tomó?, ¿ o son conjeturas suyas?” no deban ser emitidas por un jurado, puesto que con interrogantes de ese talante se pone en tela de juicio la capacidad del autor de presentar la realidad tal como la podrían percibir otras personas.

Cada investigación implica algo nuevo, ya que un problema puede abordarse desde diferentes perspectivas, en una época distinta, con otra metodología y en varios contextos. Si bien es cierto que hay resultados más esperados que otros, esto no debe opacar el papel de los evaluadores de los trabajos de grado. El contexto educativo debe ser un ejemplo de espacio en el cual se reconozca fielmente lo que arroja cada investigación, más allá de los gustos o las expectativas de quienes interfieren en ella. Por eso, el investigador, ya sea en su papel de autor, director o jurado, se desprende de prejuicios y lucha contra la tentación de unirse a las ideas compartidas, que muchas veces son las más aplaudidas. No en vano, Bustamante expresa que "hay especialistas en decir lo que está de moda decir. Pero también hay una alternativa que, en la misma dirección, es sin embargo más compleja: hacer decir lo que está de moda decir, a una investigación que dice otra cosa”. Este es un obstáculo que puede superarse si se destaca el carácter innovador de la investigación y la actitud crítica del autor.

Actualmente, en algunas universidades existe la posibilidad de que un estudiante de pregrado evite la realización de una monografía si aprueba, con un promedio específico, el primer semestre de un posgrado. Cuando esto sucede, algunos docentes manifiestan peyorativamente que se está dejando de lado la investigación, sin tener en cuenta que la costumbre es la encargada de espantar a los estudiantes en este aspecto. Esto lo comparte Álvarez-Gayou (601), quien afirma: "A través del contacto con muchas y muchos alumnos de posgrado he recibido testimonios vividos de cómo varios docentes de investigación se han encargado de hacer que sus alumnos(as) aborrezcan la investigación o en el menor de los casos le tengan temor". Cuando los estudiantes no sienten ese temor y se arriesgan, optan por lo que consideran buenas alternativas. En este caso, una buena decisión no tiene que ver con la elaboración de un trabajo de grado que los llene y les dé placer académico, sino con un trabajo que les permita graduarse rápido porque no cuentan con las comodidades financieras como para pagar un semestre de posgrado.

En relación con este asunto, Bustamante, al hablar del pragmatismo como el tercer paradigma de recontextualización de la investigación, asevera que

se deduce fácilmente qué habría qué enseñar: toda metodología que lleve a lo útil. Ello ya no para ahorrar dificultad, sino para ahorrar tiempo. Con quién se gradúa uno de manera más fácil, en qué universidad permiten hacer una tesis entre varios, qué tema le gustaría a este profesor... son consideraciones que ponen al sujeto entre paréntesis, pues lo que haga no lo implica, no lo compromete, no lo transforma. (17)

Con respecto a este asunto, hay dos posibles roles para un profesor: el que muestra la investigación como un ejercicio aburridor y tenebroso o quien se encarga de orientar $y$, desde la perspectiva como jurado evaluador, valorar cada investigación por lo que se propone y se destaca y no por lo que hace falta.

Volviendo al caso específico de la investigación lingüística, se encuentra, por ejemplo, la posibilidad de que se limite demasiado en sus propósitos. No es ajeno a la realidad que investigaciones dirigidas esencialmente al estudio de un fenómeno lingüístico, al ser sustentadas tengan que enfrentarse a la pregunta cliché: “¿Y cuál es el aporte o impacto pedagógico de este trabajo?" La respuesta sería algo como: "ninguno, pues se trata de una investigación lingüística”. De todas formas, en un caso hipotético de este talante, lo más común sería que el estudiante investigador no responda eso, pues su nota está en juego, sino que junte los nervios y al afán para responder algo que se pueda tomar como un aporte pedagógico de su trabajo, y se siente 
obligado a rebuscarse para darle gusto al jurado que lo haya cuestionado al respecto. Por eso se debe destacar no solamente el papel de los orientadores o directores, sino también el de todos aquellos que de manera directa o indirecta intervienen en el desarrollo de una investigación.

\section{Las correcciones en la investigación}

Finalmente, otra de las encrucijadas que deben enfrentar los estudiantes durante su vida como tesistas tiene que ver con las correcciones. Es innegable que cada jurado tiene su punto de vista y parte de unos saberes previos a la hora de evaluar un trabajo; esto lleva a que no esté de acuerdo con algunos aportes de la investigación que esté revisando, razón por la cual sugiere cambios o replanteamientos en diversos aspectos. Por otro lado, esa fundamentación teórica de los jurados también se presta para que ultracorrija, ya que es improbable que alguien lo sepa todo y esté actualizado en todos los temas. Por consiguiente, el estudiante investigador se enfrenta al siguiente dilema: si le hace caso al jurado, daña el trabajo que ha elaborado con tanta dedicación, y si no le hace caso, la nota bajará. Esto incluso aplica en lo que tiene que ver con aspectos ortográficos y de redacción, pues un jurado ultracorrige cuando no se actualiza; por ejemplo, de acuerdo con la Ortografía Básica de la Lengua Española, publicada en el año 2012 por la Real Academia Española, a la palabra "solo" y a los demostrativos en función de pronombre ya no se les marca tilde, pero aún hay muchas personas que lo hacen, puesto que así lo prescribían normas anteriores.

De todas formas, volviendo al tema de la evaluación de las investigaciones, si el jurado no se actualiza y se rige por el manual de ortografía de su preferencia, entonces será el antagonista de uno de los muchos casos en los que infortunadamente el estudiante investigador está en lo correcto, pero el jurado tiene la razón, aunque no la tenga. En consecuencia, se recomienda que toda investigación sea revisada con ojos nuevos y que se permita el cambio de paradigmas y la actualización del conocimiento, en el ámbito académico que siempre propugna por la actitud crítica, pero que no siempre exhorta a que se escuchen voces distintas de las clásicas.

Como conclusión, es válido pensar en la investigación como una actividad intelectual que lleva a la actualización del conocimiento. Para que esta actitud se propague, es necesario que tanto los investigadores como los directores y jurados no limiten su papel al se- guimiento fiel de normas, reglas, parámetros y costumbres. Kuhn (132), en su ensayo en el que prevalece la idea de los paradigmas, aclara: "En la ciencia un paradigma rara vez es un objeto que se pueda replicar"; sin embargo, es común ver que, en el ámbito académico, las investigaciones se ciñen a lo que muchos consideran que son las únicas opciones paradigmáticas por seguir. Pareciera como si los mismos paradigmas o modelos de investigación fueran universales e indiscutibles, y no se tiene en cuenta que una investigación, basada en su problema, sus objetivos y la época en la que está inscrita, puede guiarse por una metodología particular y que sea la más adecuada, a pesar de que no sea expuesta o defendida por un autor clásico.

\section{Referencias}

Álvarez-Gayou, Juan Luis. "Los métodos mixtos y la docencia en investigación”. Metodología de la investigación. Eds. Roberto Hernández Sampieri, Carlos Fernández y María del Pilar Baptista. México: McGraw-Hill, 2010. 601. Impreso.

Benites, Miguel. "Los investigadores opinan". Metodología de la investigación. Ed. Roberto Hernández Sampieri, Carlos Fernández y María del Pilar Baptista. México: McGraw-Hill, 2010. 169. Impreso.

Beuchot, Mauricio. La retórica como pragmática y hermenéutica. Barcelona: Anthropos Editorial, 1998. Impreso.

Bunge, Mario. La ciencia: su método y su filosofía. Buenos Aires: Siglo Veinte, 1960.

Bustamante, Guillermo. La investigación visible desde aquí. Cuatro paradigmas de recontextualización. Web. 2013.

Camacho, Briceida. Metodología de la investigación científica. Tunja: Universidad Pedagógica y Tecnológica de Colombia, 2003. Impreso.

Cruz, Roberto. "Los investigadores opinan”. Metodología de la investigación. Ed. Roberto Hernández Sampieri, Carlos Fernández y María del Pilar Baptista. México: McGraw-Hill, 2010. 343-4. Impreso.

Kuhn, Thomas. La estructura de las revoluciones científicas. México, D. F.: Fondo de Cultura Económica, 2013. Impreso.

Mora, Paulina de la. “¿Por qué es importante que los niños y jóvenes aprendan a investigar?” Metodología de la investigación. Ed. Roberto Hernández Sampieri, Carlos Fernández y María del Pilar Baptista. México: McGrawHill, 2010. 359. Impreso.

Tena, Antonio. "Los investigadores opinan”. Metodología de la investigación. Eds. Roberto Hernández Sampieri, Carlos Fernández y María del Pilar Baptista. México: McGraw-Hill, 2010. 487-8. Impreso. 